\title{
Analytic study of MHD flow and boundary layer control over a non- isothermal flat plate
}

\author{
M. Shanmugapriya * \\ Department of Mathematics, SSN College of Engineering, Chennai, India
}

\section{A RT ICLE INFO}

\section{Article history}

Received 16 December 2016

Received in revised form

12 February 2017

Accepted 20 February 2017

\section{Keywords:}

Gyarmati's variational principle

MHD boundary layer

Heat transfer

Non-isothermal

\begin{abstract}
A B S T R A C T
The objective of the present work is to investigate the boundary layer flow and heat transfer over a moving flat plate in the presence of a transverse magnetic field. Gyarmati's variational principle developed on the thermodynamic theory of irreversible processes is employed to solve the problem numerically. The governing boundary layer equations are approximated as simple polynomial functions, and the functional of the variational principle is constructed. The Euler-Langrange equations are reduced to simple polynomial equations in terms of boundary layer thicknesses. The velocity and temperature as well as skin friction and heat transfer parameters are analysed for any given values of Prandtl number $P_{r}$, magnetic parameter $M$, Eckert number $E c$, moving parameter $\varepsilon$ and $n$. The obtained numerical solutions are compared with known numerical solutions and the comparison is found to be satisfactory.
\end{abstract}

(C) 2017 The Authors. Published by IASE. This is an open access article under the CC BY-NC-ND license (http://creativecommons.org/licenses/by-nc-nd/4.0/).

\section{Introduction}

The study of boundary layer flow of an incompressible electrically conducting viscous fluid on a moving surface has an important bearing on several technical applications, such as in metallurgy and chemical processes industries. Blasius (1908) was the first to investigate and presented a theoretical result for the boundary layer flow over a flat plate in a uniform stream. A numerical investigation of the classical Blasius for the equation flat plate problem was presented by Cortell (2005). Sakiadis (1961) considered the problem of forced convection along an isothermal moving plate. Erickson et al. (1966) extended this problem to study the temperature distribution in the boundary layer when the sheet is maintained at a constant temperature with suction or blowing. An experimental and theoretical treatment was made by Tsou et al. (1967).

Ishak et al. (2011) studied the steady laminar boundary layer flow over a moving plate in moving fluid with convective surface boundary condition and in the presence of thermal radiation. In this problem they combine two problems (i.e.) Blasius flow and Sakiadis flow using the composite velocity

\footnotetext{
* Corresponding Author.

Email Address: shanmugapriyam@ssn.edu.in https://doi.org/10.21833/ijaas.2017.04.011

2313-626X/C) 2017 The Authors. Published by IASE.

This is an open access article under the CC BY-NC-ND license

(http://creativecommons.org/licenses/by-nc-nd/4.0/)
}

$U=U_{w}+U_{\infty}$ introduced by Afzal et al. (1933). By using this composite velocity, we are able to investigate the flow characteristics when both the plate and the fluid are in moving conditions. Kumari and Nath (2001) studied the problem of MHD boundary layer flow of a non-newtonian fluid over a continuously moving surface with a parallel free stream. Recently, Jat and Neemawat (2014) discussed MHD boundary layer flow and heat transfer over a moving non-isothermal flat plate.

The object of the present paper is to study the boundary layer flow and heat transfer for an electrically conducting viscous fluid on a moving flat plate in the presence of a transverse magnetic field by using Gyarmati's variational technique. This technique is one of the most general and exact variational technique in solving flow and heat transfer problems. Chandrasekar (2003) and Shanmugapriya and Chandrasekar (2008) already applied this technique for steady and unsteady heat transfer and boundary layer flow problems.

\section{Mathematical formulation}

The system of steady, two-dimensional, laminar boundary layer flow of a viscous incompressible electrically conducting fluid over a non-isothermal flat plate is considered. The $\mathrm{x}$-axis is taken along the plate and the $y$-axis is normal to it. In this study it is assumed that plate has a constant velocity $U_{w}$, in the same or opposite direction to the free stream $U_{\infty}$. A uniform magnetic field of strength $B_{0}$ is applied 
normal to the x-axis. The magnetic Reynolds number is taken to be small, therefore the induced magnetic field is neglected. Under these assumptions, the boundary layer equations are Eqs. 1-3:

$\frac{\partial u}{\partial x}+\frac{\partial v}{\partial y}=0$

$u \frac{\partial u}{\partial x}+v \frac{\partial u}{\partial y}=\vartheta \frac{\partial^{2} u}{\partial y^{2}}-\frac{\sigma_{e} B_{0}^{2} u^{2}}{\rho}$

$u \frac{\partial T}{\partial x}+v \frac{\partial T}{\partial y}=\left(\frac{\mathrm{K}}{\rho c_{p}}\right) \frac{\partial^{2} T}{\partial y^{2}}+\left(\frac{\mu}{\rho c_{p}}\right)\left(\frac{\partial u}{\partial y}\right)^{2}+\frac{\sigma_{e} B_{0}^{2} u^{2}}{\rho c_{p}}$

where $\mathrm{u}, \mathrm{v}$ are the velocity components of the fluid in the $x$ and $y$-directions respectively, $T$ is the temperature of the fluid, $\mathrm{T}_{\mathrm{w}}$ is the wall temperature, $T_{\infty}$ is the free stream temperature, $\vartheta$ is the kinematic viscosity, $\rho$ is the fluid density, $\kappa$ is the thermal conductivity of the fluid, $c_{p}$ is the specific heat at constant pressure of the fluid, $\mu$ is the coefficient of viscosity, $\sigma_{e}$ is the electrical conductivity, It is assumed that the wall temperature $T_{w}$ is greater than the free stream temperature $T_{\infty}$.

The initial and boundary conditions of the system are (Eq. 4):

$y=0 \Rightarrow u=U_{w}, v=0, T=T_{w}$

$y \rightarrow 0 \Rightarrow u=U_{\infty}$ (const), $T \rightarrow T_{\infty}$.

The wall temperature $T_{w}$ and the free-stream temperature $T_{\infty}$ satisfy the power law (Eq. 5):

$T_{w}-T_{\infty}=A x^{n}$

\section{Variational formulation of the problem}

The purpose of this analysis is to obtain the approximate numerical solution of irreversible thermodynamics problem by a variational technique. Gyarmati (1969, 1979) introduced a genuine variational principle called the "Governing Principle of Dissipative Processes" (GPDP) which is given in its universal form (Eq. 6):

$\delta \int_{V}[\sigma-\Psi-\varphi] d V=0$.

The principle Eq. 6 is valid for linear, quasi-linear and certain types of non-linear transport processes at any instant of time under constraints that the balance equations are satisfied. In Eq. 6, $\sigma$ is the entropy production $\Psi$ and $\Phi$ are dissipation potentials and $\mathrm{V}$ is the total volume of the thermodynamic system. In the Eq. 7,

$\rho \dot{a}_{\iota}+\nabla \cdot \vec{\jmath}_{l}=\sigma_{i} \quad(i=1,2,3 \ldots \ldots f)$

$\overrightarrow{\jmath_{l}}$ is the flux and $\sigma \mathrm{i}$ is the source density of the $\mathrm{i}^{\text {th }}$ extensive transport quantity ai. $\sigma$ can always be written in the bilinear form as Eq. 8:

$\sigma=\sum_{i=1}^{f} \overrightarrow{J_{l}} \cdot \overrightarrow{X_{l}} \geq 0$ where, $\vec{J}_{i}$ and $\vec{X}_{i}$ are fluxes and forces respectively. According to Onsager $(1931 \mathrm{a}, \mathrm{b})$ linear theory the fluxes are linear functions, that are Eqs. 9 and 10:

$\overrightarrow{J_{l}}=\sum_{k=1}^{f} L_{i k} \overrightarrow{X_{k}}, \quad(i=1,2,3 \ldots . f)$

or alternatively

$\overrightarrow{X_{l}}=\sum_{k=1}^{f} R_{i k} \overrightarrow{J_{k}}, \quad(i=1,2,3 \ldots . f)$.

The constants $\mathrm{L}_{\mathrm{ik}}$ and $\mathrm{R}_{\mathrm{ik}}$ are conductivities and resistances respectively and they satisfy the reciprocal relations (Eq. 11):

$L_{i k}=L_{k i}$ and $R_{i k}=R_{k i},(i, k=1,2,3, \ldots f)$.

The matrices of $\mathrm{Lik}_{\mathrm{ik}}$ and $\mathrm{R}_{\mathrm{ik}}$ are mutual reciprocals. That is Eq. 12:

$\sum_{m=1}^{f} L_{i m} R_{m k}=\sum_{m=1}^{f} L_{m k} R_{i m}=\delta_{i k} \quad(i, k=$ $1,2,3 \ldots . . f)$

where, $\delta_{\mathrm{ik}}$ is the Kronecker delta. The local dissipation potentials $\Psi$ and $\Phi$ are defined as Eqs. 13 and 14:

$\Psi(\overrightarrow{\mathrm{X}}, \overrightarrow{\mathrm{X}})=(1 / 2) \sum_{i, k=1}^{f} L_{i k} \overrightarrow{X_{l}}, \overrightarrow{X_{k}} \geq 0$,

$\varphi(\overrightarrow{\mathrm{J}}, \overrightarrow{\mathrm{J}})=(1 / 2) \sum_{i, k=1}^{f} R_{i k} \overrightarrow{J_{l},} \overrightarrow{J_{k}} \geq 0$,

since in the case of transport processes $\overrightarrow{X_{l}}$ can be generated as gradients of certain " $\Gamma$ " variable, it is written as Eq. 15:

$\vec{X}_{i}=\nabla \Gamma_{i}$

The principle (Eq. 6) with the help of Eqs. 8, 11, 13, 14 and 15, takes the form Eq. 16:

$$
\begin{aligned}
& \delta \int_{V}\left[\sum_{i=1}^{f} \overrightarrow{J_{l}} \cdot \nabla \Gamma_{i}-(1 / 2) \sum_{i, k=1}^{f} L_{i k} \nabla \Gamma_{i} \cdot \nabla \Gamma_{k}-\right. \\
& \left.(1 / 2) \sum_{i, k=1}^{f} R_{i k} \overrightarrow{J_{l}} \cdot \overrightarrow{J_{k}}\right] d V=0 .
\end{aligned}
$$

The principle (Eq. 6) is also in energy picture as (Eq. 17):

$\delta \int_{V}\left[T \sigma-\Psi^{*}-\varphi^{*}\right] d V=0$.

here, $T \sigma$ is the energy dissipation and the dissipation potentials $\Psi^{*}$ and $\Phi^{*}=$ are given by Eq. 18 :

$\Psi^{*}=\mathrm{T} \Psi$ and $\Phi^{*}=\mathrm{T} \Phi$.

It is found that GPDP in energy picture given by Eq. 17 is always advantageous for dealing with thermo hydrodynamic systems. This variational principle has been already applied for various dissipative systems and was established as the most general and exact principle of macroscopic continuum physics.

The balance equations of the system play a central role in the formulation of Gyarmati's variational principle and hence the governing Eqs. 1, 
2 and 3 are written in the balance forms as Eqs. 19, 20 and 21

$\nabla \cdot \vec{V}=0,(\vec{V}=i u+j v)$

$\rho(\vec{V} . \nabla) \vec{V}+\nabla \cdot \overline{\bar{P}}=0$,

$\rho c_{\mathrm{p}}(\vec{V} \cdot \nabla) T+\nabla \cdot \overrightarrow{J_{q}}=\mu\left(u_{y}^{2}\right)+\left(\sigma_{e} B_{0}^{2} u^{2}\right)$.

These equations represent the mass, momentum and energy balances respectively. In Eq. $20 \overline{\bar{p}}$ denotes the pressure tensor which can be decomposed as Eq. 22:

$\overline{\bar{p}}=p \overline{\bar{\delta}}+\stackrel{\circ}{{ }_{\bar{p}}^{v s}}$

where, $\mathrm{p}$ is the hydrostatic pressure, $\overline{\bar{\delta}}$ is the unit tensor, and ${ }_{\overline{\bar{p}}}^{{ }^{v s}}$ is the symmetrical part of the viscous pressure tensor, whose trace is zero. In the energy picture, the energy dissipation for the present system is given by Eq. 22:

$T \sigma=-J_{q}(\partial \ln T / \partial y)-P_{12}(\partial u / \partial y)$.

The heat flux $J_{q}$ and $P_{12}$ the only component of momentum flux $\overline{\bar{p}}^{\text {vs }}$ satisfy the constitutive relations connecting the independent fluxes and forces as Eq. 22:

$J_{q}=-L_{\lambda}\left(\frac{\partial \ln T}{\partial y}\right)$ and $P_{12}=-L_{s}\left(\frac{\partial u}{\partial y}\right)$,

here, $\mathrm{L}_{\lambda}=\lambda \mathrm{T}$ and $\mathrm{L}_{s}=\mu$ where $\lambda$ and $\mu$ are the thermal conductivity and viscosity respectively. It is well known that $\ln \mathrm{T}$ is the proper state variable instead of $\mathrm{T}$ when the principle assumes energy picture (Eq. 19). With the help of Eq. 24 the dissipation potentials in the energy picture are found as follows (Eqs. 25 and 26):

$\Psi^{*}=(1 / 2)\left[L_{\lambda}(\partial \ln T / \partial y)^{2}+L_{S}(\partial u / \partial y)^{2}\right]$

$\varphi^{*}=(1 / 2)\left[R_{\lambda} J_{q}^{2}+R_{S} P_{12}^{2}\right]$

where, $L_{\lambda}=R_{\lambda}^{-1}$ and $L_{s}=R_{s}^{-1}$. Using the Eqs. 23, 25 and 26 Gyarmati's variational principle in the energy picture (Eq. 17) is formulated as:

$\delta \int_{0}^{l} \int_{0}^{\infty}\left[\begin{array}{c}-J_{q}\left(\frac{\partial \ln T}{\partial y}\right)-P_{12}\left(\frac{\partial u}{\partial y}\right)- \\ \left(\frac{L_{\lambda}}{2}\right)\left(\frac{\partial \ln T}{\partial y}\right)^{2}-\left(\frac{L_{s}}{2}\right)\left(\frac{\partial u}{\partial y}\right)^{2}- \\ \left(\frac{R_{\lambda}}{2}\right) J_{q}^{2}-\left(\frac{R_{s}}{2}\right) P_{12}^{2}\end{array}\right] d y d x=0$,

in which ' $\mathrm{l}$ ' is the representative length of the surface.

\section{Solution procedure}

The velocity and temperature fields inside the respective boundary layers are approximated as a fourth degree polynomial function:

$$
\begin{aligned}
& \frac{\left(u-U_{w}\right)}{\left(U_{\infty}-U_{w}\right)}=\frac{2 y}{d_{1}}-\frac{2 y^{3}}{d_{1}^{3}}+\frac{y^{4}}{d_{1}^{4}},\left(y<d_{1}\right) \\
& u=U_{\infty},\left(y \geq d_{1}\right) \\
& \frac{\left(T-T_{\infty}\right)}{\left(T_{0}-T_{\infty}\right)}=\theta=1-\frac{2 y}{d_{2}}+\frac{2 y^{3}}{d_{2}^{3}}+\frac{y^{4}}{d_{2}^{4}},\left(y<d_{2}\right) \\
& T=T_{\infty},\left(y \geq d_{2}\right)
\end{aligned}
$$

where, $d_{1}$ and $d_{2}$ are hydrodynamical and thermal boundary layer thicknesses respectively. The velocity and thermal profiles (Eq. 28) satisfy the following compatibility conditions (Eq. 29):

$y=0 ; u=U_{w}, v=0, \frac{\partial T}{\partial y}=0($ smoothfit $), \frac{\partial^{2} u}{\partial y^{2}}=0$,

$y=d_{1} ; u=U_{\infty}, \frac{\partial u}{\partial y}=0($ smoothfit $), \frac{\partial^{2} u}{\partial y^{2}}=0$,

$y=d_{2} ; T=T_{\infty}, \frac{\partial T}{\partial y}=0$ (smoothfit),$\frac{\partial^{2} T}{\partial y^{2}}=0$.

Using the boundary conditions (Eq. 29), the transverse velocity component $\mathrm{v}$ is obtained from the mass balance Eq. 1 as Eq. 30:

$v=\left(U_{\infty}-U_{w}\right)\left[\frac{y^{2}}{d_{1}^{2}}-\frac{3 y^{4}}{2 d_{1}^{4}}+\frac{4 y^{5}}{5 d_{1}^{5}}\right] d_{1}^{\prime}$.

To formulate Gyarmati's variational principle the velocity and temperature functions (Eq. 28) are substituted in the momentum and energy balance Eqs. 2 and 3, and on direct integration with respect to $y$ with the help of smooth fit boundary conditions $\frac{\partial u}{\partial y}=0$ and $\frac{\partial T}{\partial y}=0$ the fluxes $\mathrm{P}_{12}$ and Jq are obtained respectively as given below (Eq. 31):

$-\frac{P_{12}}{L_{s}}=\left[\frac{\left(U_{\infty}-U_{w}\right)^{2} d_{1}^{\prime}}{\vartheta}\right]\left[0.11746031-\frac{2 y^{3}}{3 d_{1}^{3}}+\frac{7 y^{5}}{5 d_{1}^{5}}-\frac{11 y^{6}}{15 d_{1}^{6}}-\right.$ $\left.\frac{3 y^{7}}{7 d_{1}^{7}}+\frac{2 y^{8}}{5 d_{1}^{8}}-\frac{4 y^{9}}{45 d_{1}^{9}}\right]+\left[\frac{U_{w}\left(U_{\infty}-U_{w}\right) d_{1}^{\prime}}{\vartheta}\right]\left[0.3-\frac{y^{2}}{d_{1}^{2}}+\frac{3 y^{4}}{2 d_{1}^{4}}+\frac{4 y^{5}}{5 d_{1}^{5}}\right]+$ $\left[\frac{\left(U_{\infty}-U_{w}\right) \sigma_{e} B_{0}^{2}}{\vartheta \rho}\right]\left[-0.7 d_{1}+\frac{y^{2}}{d_{1}^{2}}-\frac{y^{4}}{2 d_{1}^{4}}+\frac{y^{5}}{5 d_{1}^{5}}\right]+$

$\left[\frac{\sigma_{e} B_{0}^{2} U_{w}}{\vartheta \rho}\right]\left[-d_{1}+y\right],\left(0 \leq y \leq d_{1}\right)$

$-\frac{J_{q}}{L_{\lambda}}=\left[\frac{n P_{r}\left(U_{\infty}-U_{w}\right)\left(T_{w}-T_{\infty}\right)}{\vartheta x}\right]\left[-0.7 d_{1}+0.86667 \frac{d_{1}^{2}}{d_{2}}-\right.$

$0.47857 \frac{d_{1}^{4}}{d_{2}^{3}}+0.19444 \frac{d_{1}^{5}}{d_{2}^{4}}+\frac{y^{2}}{d_{1}}-\frac{4 y^{3}}{3 d_{1} d_{2}}+\frac{4 y^{5}}{5 d_{1} d_{2}^{3}}-\frac{y^{6}}{3 d_{1} d_{2}^{4}}-$

$\left.\frac{y^{4}}{2 d_{1}^{3}}+\frac{4 y^{5}}{5 d_{1}^{3} d_{2}}-\frac{4 y^{7}}{7 d_{1}^{3} d_{2}^{3}}+\frac{y^{8}}{4 d_{1}^{3} d_{2}^{4}}+\frac{y^{5}}{5 d_{1}^{4}}-\frac{y^{6}}{3 d_{1}^{4} d_{2}}+\frac{y^{8}}{4 d_{1}^{4} d_{2}^{3}}-\frac{y^{9}}{9 d_{1}^{4} d_{2}^{4}}\right]+$ $\left[\frac{P_{r}\left(U_{\infty}-U_{w}\right)\left(T_{w}-T_{\infty}\right)}{\vartheta}\right] d_{2}^{\prime}\left[-0.8666 \frac{d_{1}^{2}}{d_{2}^{2}}+1.43571 \frac{d_{1}^{4}}{d_{2}^{4}}-\right.$

$0.77778 \frac{d_{1}^{5}}{d_{2}^{5}}+\frac{4 y^{3}}{3 d_{1} d_{2}^{2}}-\frac{12 y^{5}}{5 d_{1} d_{2}^{4}}+\frac{4 y^{6}}{3 d_{1} d_{2}^{5}}-\frac{4 y^{5}}{5 d_{1}^{3} d_{2}^{2}}+\frac{12 y^{7}}{7 d_{1}^{3} d_{2}^{4}}-\frac{y^{8}}{d_{1}^{3} d_{2}^{5}}+$ $\left.\frac{y^{6}}{3 d_{1}^{4} d_{2}^{2}}-\frac{3 y^{8}}{4 d_{1}^{4} d_{2}^{4}}+\frac{4 y^{9}}{9 d_{1}^{4} d_{2}^{5}}\right]+\left[\frac{P_{r}\left(U_{\infty}-U_{w}\right)\left(T_{w}-T_{\infty}\right)}{\vartheta}\right] d_{1}^{\prime}\left[0.33333 \frac{d_{1}}{d_{2}}-\right.$ $0.51429 \frac{d_{1}^{3}}{d_{2}^{3}}+0.27222 \frac{d_{1}^{4}}{d_{2}^{4}}-\frac{2 y^{3}}{3 d_{1}^{2} d_{2}}+\frac{6 y^{5}}{5 d_{1}^{2} d_{2}^{3}}-\frac{2 y^{6}}{3 d_{1}^{2} d_{2}^{4}}+$ $\left.\frac{3 y^{5}}{5 d_{1}^{4} d_{2}}-\frac{9 y^{7}}{7 d_{1}^{4} d_{2}^{3}}+\frac{3 y^{8}}{4 d_{1}^{4} d_{2}^{4}}-\frac{4 y^{6}}{15 d_{1}^{5} d_{2}}+\frac{3 y^{8}}{5 d_{1}^{5} d_{2}^{3}}-\frac{16 y^{9}}{45 d_{1}^{4} d_{2}^{4}}\right]+$ $\left[\frac{n P_{r} U_{w}\left(T_{w}-T_{\infty}\right)}{\vartheta x}\right]\left[-d_{1}+\frac{d_{1}^{2}}{d_{2}}-\frac{d_{1}^{4}}{2 d_{2}^{3}}+\frac{d_{1}^{5}}{5 d_{2}^{4}}+y-\frac{y^{2}}{d_{2}}+\right.$ $\left.\frac{y^{4}}{2 d_{2}^{3}}-\frac{y^{5}}{5 d_{2}^{4}}\right]+\left[\frac{P_{r} U_{w}\left(T_{w}-T_{\infty}\right)}{\vartheta}\right] d_{2}^{\prime}\left[-\frac{d_{1}^{2}}{d_{2}^{2}}+\frac{3 d_{1}^{4}}{2 d_{2}^{4}}-\frac{4 d_{1}^{5}}{5 d_{2}^{4}}+\right.$ $\left.\frac{y^{2}}{d_{2}^{2}}-\frac{3 y^{4}}{2 d_{2}^{4}}+\frac{4 y^{5}}{5 d_{2}^{5}}\right]-\left[\frac{P_{r}\left(U_{\infty}-U_{w}\right)^{2}}{c_{p}}\right]\left[-\frac{1.48571}{d_{1}}+\frac{4 y}{d_{1}^{2}}+\frac{36 y^{5}}{5 d_{1}^{6}}+\right.$ $\left.\frac{16 y^{7}}{7 d_{1}^{8}}-\frac{24 y^{3}}{3 d_{1}^{4}}+\frac{16 y^{4}}{4 d_{1}^{5}}-\frac{48 y^{6}}{6 d_{1}^{7}}\right]-$ $\left[\frac{P_{r} \sigma_{e} B_{0}^{2}\left(U_{\infty}-U_{w}\right)^{2}}{\vartheta \rho c_{p}}\right]\left[-0.58284 d_{1}+\frac{4 y^{3}}{3 d_{1}^{2}}+\frac{4 y^{7}}{7 d_{1}^{6}}+\frac{y^{9}}{9 d_{1}^{8}}-\frac{8 y^{5}}{5 d_{1}^{4}}+\right.$ $\left.\frac{4 y^{6}}{6 d_{1}^{5}}-\frac{4 y^{8}}{8 d_{1}^{7}}\right]-\left[\frac{P_{r} \sigma_{e} B_{0}^{2} U_{w}^{2}}{\vartheta \rho c_{p}}\right]\left[y-d_{1}\right]-$ 
$\left[\frac{P_{r} \sigma_{e} B_{0}^{2} U_{w}\left(U_{\infty}-U_{w}\right)}{\vartheta \rho c_{p}}\right]\left[-1.4 d_{1}+\frac{2 y^{2}}{d_{1}}-\frac{y^{4}}{d_{1}^{3}}+\frac{2 y^{5}}{5 d_{1}^{4}}\right],(0 \leq y \leq$

$\left.d_{1}\right) ; P_{r} \leq 1$

$-\frac{J_{q}}{L_{\lambda}}=\left[\frac{n P_{r}\left(U_{\infty}-U_{w}\right)\left(T_{w}-T_{\infty}\right)}{\vartheta x}\right]\left[-0.3 d_{2}+y-\frac{y^{2}}{d_{2}}+\frac{y^{4}}{2 d_{2}^{3}}-\frac{y^{5}}{5 d_{2}^{4}}\right]+$ $\left[\frac{P_{r} U_{\infty}\left(T_{w}-T_{\infty}\right)}{\vartheta}\right]\left[-0.3+\frac{y^{2}}{d_{2}^{2}}-\frac{3 y^{4}}{2 d_{2}^{4}}+\frac{4 y^{5}}{5 d_{1}^{5}}\right]+\left[\frac{P_{r}\left(U_{\infty}-U_{w}\right)^{2}}{c_{p}}\right]\left[\frac{4 d_{2}}{d_{1}^{2}}+\right.$ $\frac{8 d_{2}^{3}}{d_{1}^{4}}-\frac{4 d_{2}^{4}}{d_{1}^{5}}-\frac{36 d_{2}^{5}}{d_{1}^{6}}+\frac{8 d_{2}^{6}}{d_{1}^{7}}-\frac{16 d_{2}^{7}}{7 d_{1}^{8}}+\frac{4 y}{d_{1}^{2}}-\frac{8 y^{3}}{d_{1}^{4}}+\frac{4 y^{4}}{d_{1}^{5}}+\frac{36 y^{5}}{5 d_{1}^{6}}-$ $\left.\frac{8 y^{6}}{d_{1}^{7}}+\frac{16 y^{7}}{7 d_{1}^{8}}\right]-\left[\frac{P_{r} \sigma_{e} B_{0}^{2}\left(U_{\infty}-U_{w}\right)^{2}}{\vartheta \rho c_{p}}\right]\left[\frac{-4 d_{2}^{3}}{3 d_{1}^{2}}+\frac{8 d_{2}^{5}}{5 d_{1}^{4}}-\frac{2 d_{2}^{6}}{3 d_{1}^{5}}-\frac{4 d_{2}^{7}}{7 d_{1}^{6}}+\right.$ $\left.\frac{d_{2}^{8}}{2 d_{1}^{7}}-\frac{d_{2}^{9}}{9 d_{1}^{8}}+\frac{4 y^{3}}{3 d_{1}^{2}}+\frac{4 y^{7}}{7 d_{1}^{6}}+\frac{y^{9}}{9 d_{1}^{3}}-\frac{8 y^{5}}{5 d_{1}^{4}}+\frac{2 y^{6}}{3 d_{1}^{5}}-\frac{y^{8}}{2 d_{1}^{7}}\right]-$ $\left[\frac{P_{r} \sigma_{e} B_{0}^{2} U_{w}^{2}}{\vartheta \rho c_{p}}\right]\left[y-d_{2}\right]-\left[\frac{P_{r} \sigma_{e} B_{0}^{2} U_{w}\left(U_{\infty}-U_{w}\right)}{\vartheta \rho c_{p}}\right]\left[\frac{-2 d_{2}^{2}}{d_{1}}+\frac{d_{2}^{4}}{d_{1}^{3}}-\right.$ $\left.\frac{2 d_{2}^{5}}{5 d_{1}^{4}}+\frac{2 y^{2}}{d_{1}}-\frac{y^{4}}{d_{1}^{3}}+\frac{2 y^{5}}{5 d_{1}^{4}}\right],\left(d_{1} \leq y \leq d_{2}\right) ; P_{r} \leq 1$

$-\frac{J_{q}}{L_{\lambda}}=\left[\frac{n P_{r}\left(U_{\infty}-U_{w}\right)\left(T_{w}-T_{\infty}\right)}{\vartheta x}\right]\left[-0.13333 \frac{d_{2}^{2}}{d_{1}}+0.02143 \frac{d_{2}^{4}}{d_{1}^{3}}-\right.$ $0.00556 \frac{d_{2}^{5}}{d_{1}^{4}}+\frac{y^{2}}{d_{1}}-\frac{4 y^{3}}{3 d_{1} d_{2}}+\frac{4 y^{5}}{5 d_{1} d_{2}^{3}}-\frac{y^{6}}{3 d_{1} d_{2}^{4}}-\frac{y^{4}}{2 d_{1}^{3}}+\frac{4 y^{5}}{5 d_{1}^{3} d_{2}}-$ $\left.\frac{4 y^{7}}{7 d_{1}^{3} d_{2}^{3}}+\frac{y^{8}}{4 d_{1}^{3} d_{2}^{4}}+\frac{y^{5}}{5 d_{1}^{4}}-\frac{y^{6}}{3 d_{1}^{4} d_{2}}+\frac{y^{8}}{4 d_{1}^{4} d_{2}^{3}}-\frac{y^{9}}{9 d_{1}^{4} d_{2}^{4}}\right]+$ $\left[\frac{P_{r}\left(U_{\infty}-U_{w}\right)\left(T_{w}-T_{\infty}\right)}{\vartheta}\right] d_{2}^{\prime}-0.26667 \frac{d_{2}}{d_{1}}+\frac{d_{2}^{3}}{d_{1}^{3}}-\frac{d_{2}^{4}}{d_{1}^{4}}+\frac{4 y^{3}}{3 d_{1} d_{2}^{2}}-$ $\frac{12 y^{5}}{5 d_{1} d_{2}^{4}}+\frac{4 y^{6}}{3 d_{1} d_{2}^{5}}-\frac{4 y^{5}}{5 d_{1}^{3} d_{2}^{2}}+\frac{12 y^{7}}{7 d_{1}^{3} d_{2}^{4}}-\frac{y^{8}}{d_{1}^{3} d_{2}^{5}}+\frac{y^{6}}{3 d_{1}^{4} d_{2}^{2}}-\frac{3 y^{8}}{4 d_{1}^{4} d_{2}^{4}}+$ $\left.\frac{4 y^{9}}{9 d_{1}^{4} d_{2}^{5}}\right]+\left[\frac{P_{r}\left(U_{\infty}-U_{w}\right)\left(T_{w}-T_{\infty}\right)}{\vartheta}\right] d_{1}^{\prime}\left[0.13333 \frac{d_{2}^{2}}{d_{1}^{2}}-0.06429 \frac{d_{1}^{4}}{d_{2}^{3}}+\right.$ $0.02222 \frac{d_{2}^{5}}{d_{1}^{5}}-\frac{2 y^{3}}{3 d_{1}^{2} d_{2}}+\frac{6 y^{5}}{5 d_{1}^{2} d_{2}^{3}}-\frac{2 y^{6}}{3 d_{1}^{2} d_{2}^{4}}+\frac{3 y^{5}}{5 d_{1}^{4} d_{2}}-\frac{9 y^{7}}{7 d_{1}^{4} d_{2}^{3}}+$ $\left.\frac{3 y^{8}}{4 d_{1}^{4} d_{2}^{4}}-\frac{4 y^{6}}{15 d_{1}^{5} d_{2}}+\frac{3 y^{8}}{5 d_{1}^{5} d_{2}^{3}}-\frac{16 y^{9}}{45 d_{1}^{4} d_{2}^{4}}\right]+\left[\frac{n P_{r} U_{w}\left(T_{w}-T_{\infty}\right)}{\vartheta x}\right]\left[-0.3 d_{2}+\right.$ $\left.y-\frac{y^{2}}{d_{2}}+\frac{y^{4}}{2 d_{2}^{3}}-\frac{y^{5}}{5 d_{2}^{4}}\right]+\left[\frac{P_{r} U_{w}\left(T_{w}-T_{\infty}\right)}{\vartheta}\right] d_{2}^{\prime}\left[-0.3+\frac{y^{2}}{d_{2}^{2}}-\frac{3 y^{4}}{2 d_{2}^{4}}+\right.$ $\left.\frac{4 y^{5}}{5 d_{2}^{5}}\right]-\left[\frac{P_{r}\left(U_{\infty}-U_{w}\right)^{2}}{c_{p}}\right]\left[\frac{-4 d_{2}}{d_{1}^{2}}-\frac{36 d_{2}{ }^{5}}{5 d_{1}^{6}}-\frac{16 d_{2}{ }^{7}}{7 d_{1}^{8}}+\frac{24 d_{2}{ }^{3}}{3 d_{1}^{4}}-\frac{16 d_{2}{ }^{4}}{4 d_{1}^{5}}+\right.$ $\left.\frac{48 d_{2}{ }^{6}}{6 d_{1}^{7}}+\frac{4 y}{d_{1}^{2}}+\frac{36 y^{5}}{5 d_{1}^{6}}+\frac{16 y^{7}}{7 d_{1}^{8}}-\frac{24 y^{3}}{3 d_{1}^{4}}+\frac{16 y^{4}}{4 d_{1}^{5}}-\frac{48 y^{6}}{6 d_{1}^{7}}\right]-$ $\left[\frac{P_{r} \sigma_{e} B_{0}^{2}\left(U_{\infty}-U_{w}\right)^{2}}{\vartheta \rho c_{p}}\right]\left[\frac{-4 d_{2}{ }^{3}}{3 d_{1}^{2}}-\frac{4 d_{2}{ }^{7}}{7 d_{1}^{6}}-\frac{d_{2}{ }^{9}}{9 d_{1}^{8}}+\frac{8 d_{2}{ }^{5}}{5 d_{1}^{4}}-\frac{4 d_{2}{ }^{6}}{6 d_{1}^{5}}+\frac{4 d_{2}{ }^{8}}{8 d_{1}^{7}}+\right.$ $\left.\frac{4 y^{3}}{3 d_{1}^{2}}+\frac{4 y^{7}}{7 d_{1}^{6}}+\frac{y^{9}}{9 d_{1}^{8}}-\frac{8 y^{5}}{5 d_{1}^{4}}+\frac{4 y^{6}}{6 d_{1}^{5}}-\frac{4 y^{8}}{8 d_{1}^{7}}\right]-\left[\frac{P_{r} \sigma_{e} B_{0}^{2} U_{w}^{2}}{\vartheta \rho c_{p}}\right]\left[y-d_{2}\right]-$ $\left[\frac{P_{r} \sigma_{e} B_{0}^{2} U_{w}\left(U_{\infty}-U_{w}\right)}{\vartheta \rho c_{p}}\right]\left[\frac{-2 d_{2}{ }^{2}}{d_{1}}+\frac{d_{2}{ }^{4}}{d_{1}^{3}}-\frac{2 d_{2}{ }^{5}}{5 d_{1}^{4}}+\frac{2 d_{2}{ }^{2}}{d_{1}}-\frac{y^{4}}{d_{1}^{3}}+\right.$ $\left.\frac{2 y^{5}}{5 d_{1}^{4}}\right],\left(0 \leq y \leq d_{2}\right) ; P_{r} \geq 1$

The prime indicates the differentiation with respect to $\mathrm{x}$. The above expressions for $\mathrm{P}_{12}$ and $\mathrm{J}_{\mathrm{q}}$ are assumption that the Prandtl number $P_{r} \leq 1$ and $P_{r} \geq$ 1.

With the help of Eqs. 31-34 and 28 the GPDP given by (Eq. 27) is formulated and the integration of the Lagrangian with respect to $y$ is carried out. The variational principle, after simplification, is written in a simple form

$\delta \int_{0}^{l} L_{1}\left[d_{1}, d_{2}, d_{1}^{\prime}, d_{2}^{\prime}\right] d x=0 ; P_{r} \leq 1$

and $\delta \int_{0}^{l} L_{2}\left[d_{1}, d_{2}, d_{1}^{\prime}, d_{2}^{\prime}\right] d x=0 ; P_{r} \geq 1$

where, $\mathrm{L}_{1}$ and $\mathrm{L}_{2}$ are the Lagrangian densities of the principle.

The boundary layer thicknesses $d_{1}$ and $d_{2}$ are the independent parameters to be calculated and the Euler-Lagrange equations corresponding to these variational principles are:

$$
\begin{aligned}
& \left(\partial L_{1,2} / \partial d_{1}\right)-(d / d x)\left(\partial L_{1,2} / \partial d_{1}^{\prime}\right)=0 \\
& \text { and }\left(\partial L_{1,2} / \partial d_{2}\right)-(d / d x)\left(\partial L_{1,2} / \partial d_{2}^{\prime}\right)=0
\end{aligned}
$$

where, $\mathrm{L}_{1}, 2$ represents the Lagrangian densities $\mathrm{L}_{1}$ and $L_{2}$ respectively. The Eqs. 35 and 36 are second order ordinary differential equations in terms of $d_{1}$ and $d_{2}$ respectively. We now introducing the nondimensional boundary layer thicknesses $d_{1}^{*}$ and $d_{2}^{*}$ for solving these equations and are given by

$d_{1}=d_{1}^{*} \sqrt{\vartheta x / U}$

and $d_{2}=d_{2}^{*} \sqrt{\vartheta x / U}$

where, $U=U_{w}+U_{\infty}$ is the composite velocity.

The Euler-Lagrange equations of the transformed principle assume the simple forms

$\left(\partial L_{1,2} / \partial d_{1}^{*}\right)=0$ and $\left(\partial L_{1,2} / \partial d_{2}^{*}\right)=0 .\left(P_{r} \leq 1 ; P_{r} \geq 1\right)$

The coefficients of the Eq. 38 depend on the independent parameters $P_{r}, M, E c, \varepsilon, n$ and $R e$ where $P_{r}=\mu c_{p} / k \quad$ (Prandtl number) $M=2 \sigma_{e} x B_{0}^{2} / \rho U$ (Magnetic parameter), $E c=U^{2} / c_{p}\left(T_{w}-T_{\infty}\right.$ ) (Eckert number), $\varepsilon=U_{\infty} / U$ (Moving parameter) and $R e=$ $U x / \vartheta$ (Reynolds number).

\section{Analysis of results}

After getting the non-dimensional boundary layer thicknesses $d_{1}^{*}$ and $d_{2}^{*}$ for the given values of $\operatorname{Pr}, M, E c, \varepsilon, n$ and $R$ the velocity and temperature profiles, velocity and temperature gradients, skin friction and heat transfer values are calculated with the help of the following relations respectively:

$\eta=y \sqrt{U / \vartheta x}$,

$C_{f}=\sqrt{\frac{2}{R e}}\left[\left(-P_{12} / L_{s}\right)_{y=0} / U \sqrt{U / 2 \vartheta x}\right]$,

$N_{u l}=-\frac{x}{\left(T_{w}-T_{\infty}\right)\left(-\frac{J q}{L_{\lambda}}\right)_{y=0}}$

$C_{f}=\sqrt{\frac{2}{R e}}\left(-P_{12} / L_{s}\right)_{y=0}$,

$N_{u l}=-\sqrt{\operatorname{Re} / 2}\left[\left(-\frac{J_{q}}{L_{\lambda}}\right)_{y=0} /\left(T_{w}-T_{\infty}\right) \sqrt{U / 2 \vartheta x}\right]$

The results have been compared by work done by Blasius (1908), Sakiadis (1961), and Jat and Neemawat (2014). A good agreement is seen between the results.

Figs. 1 and 2 represents the velocity profiles for different values of the magnetic parameter $M$ when $\varepsilon=0, n=0$ and $n=2$ From these figures, it can be easily observed that the thickness of the velocity boundary layer decreases for increasing values of $M$.

The temperature profiles are shown in Figs. 3-8 respectively for various values of parameters. From these figures, it may be noted that the thickness of the thermal boundary layer increase with increasing values of the magnetic parameter $\mathrm{M}$ and Eckert number Ec, whereas it decreases with increasing values of the Prandtl number Pr. 


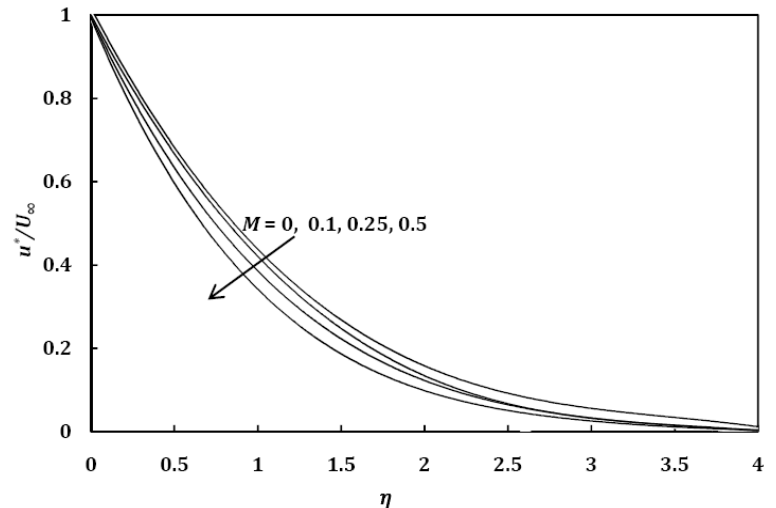

Fig. 1: Dimensionless velocity profile for different values of $\mathrm{M}$ when $\varepsilon=0$ and $\mathrm{n}=0$

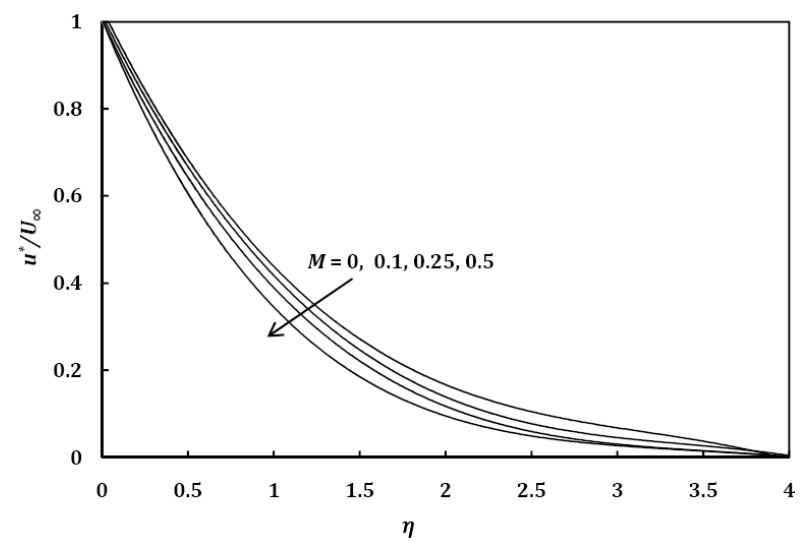

Fig. 2: Dimensionless velocity profile for different values of $\mathrm{M}$ when $\varepsilon=0$ and $\mathrm{n}=2$

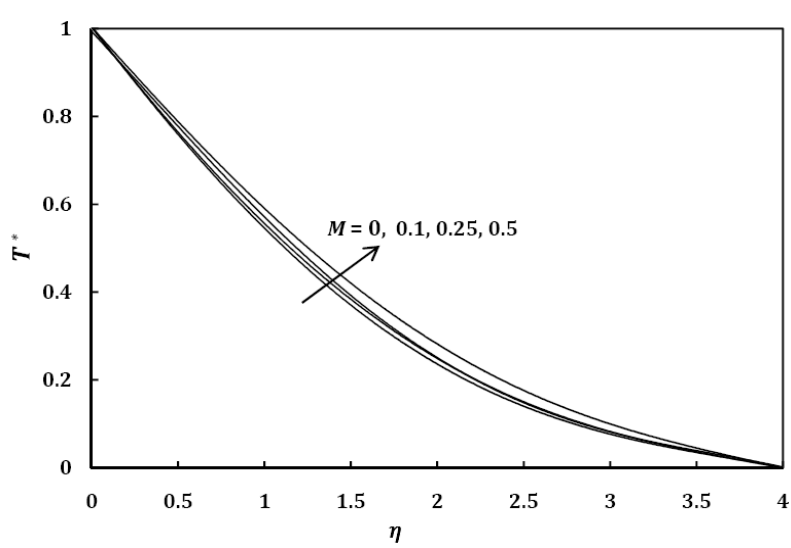

Fig. 3: Dimensionless temperature profile for different values of $\mathrm{M}$ when $\varepsilon=0.1, \mathrm{n}=0, \operatorname{Pr}=0.7$ and $\mathrm{Ec}=0.2$

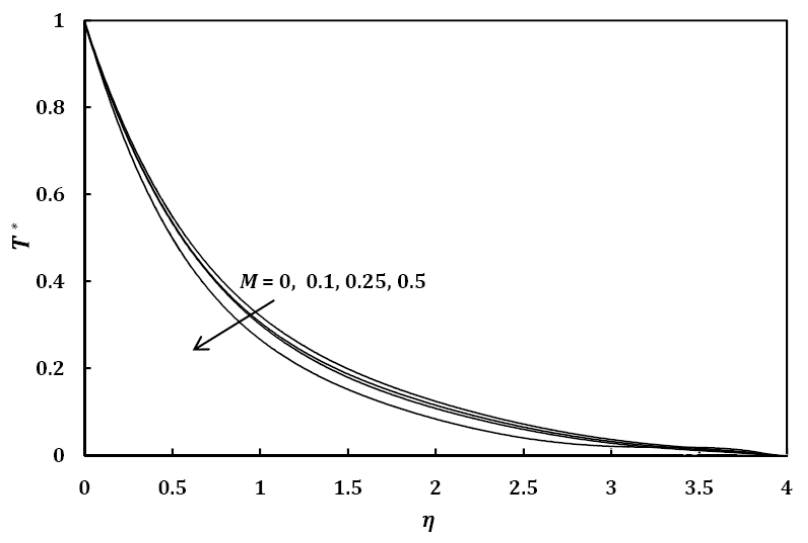

Fig. 4: Dimensionless temperature profile for different values of $\mathrm{M}$ when $\varepsilon=0.2, \mathrm{n}=2, \operatorname{Pr}=0.7$ and $\mathrm{Ec}=0.1$

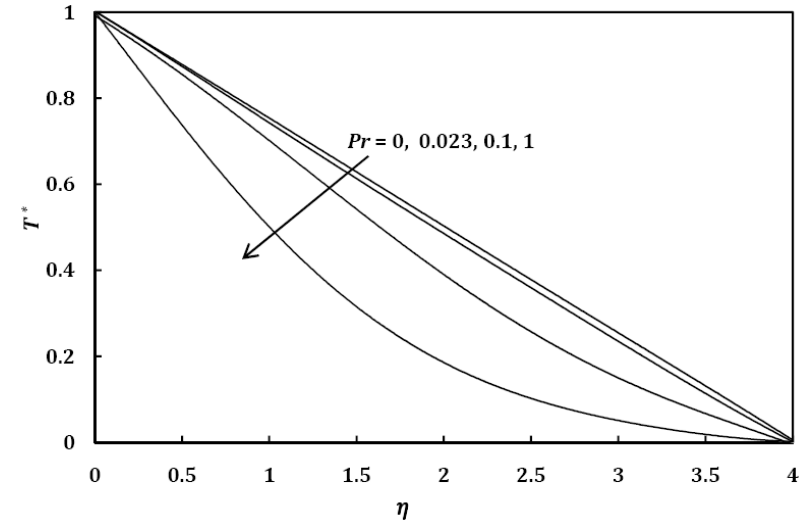

Fig. 5: Dimensionless temperature profile for different values of $\operatorname{Pr}$ when $\varepsilon=0.1, \mathrm{n}=0, \mathrm{M}=0.25$ and $\mathrm{Ec}=0.2$

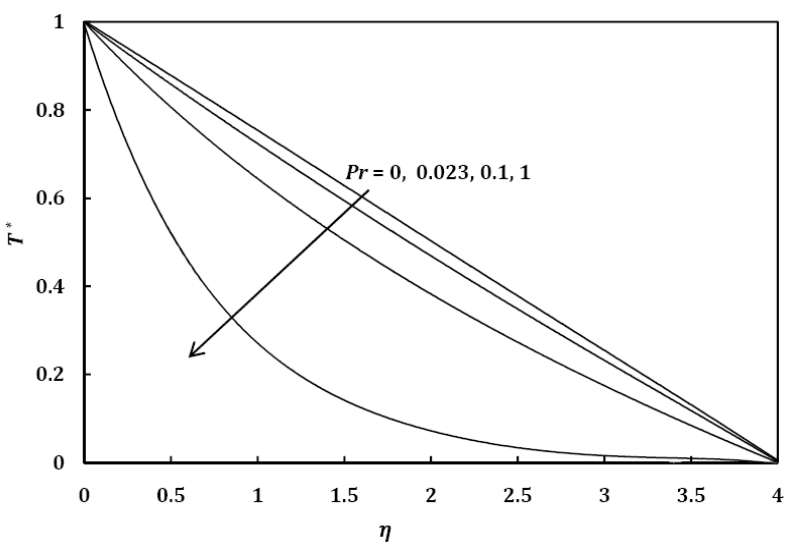

Fig. 6: Dimensionless temperature profile for different values of $\operatorname{Pr}$ when $\varepsilon=0.2, n=2, M=0.5$ and $E c=0.1$

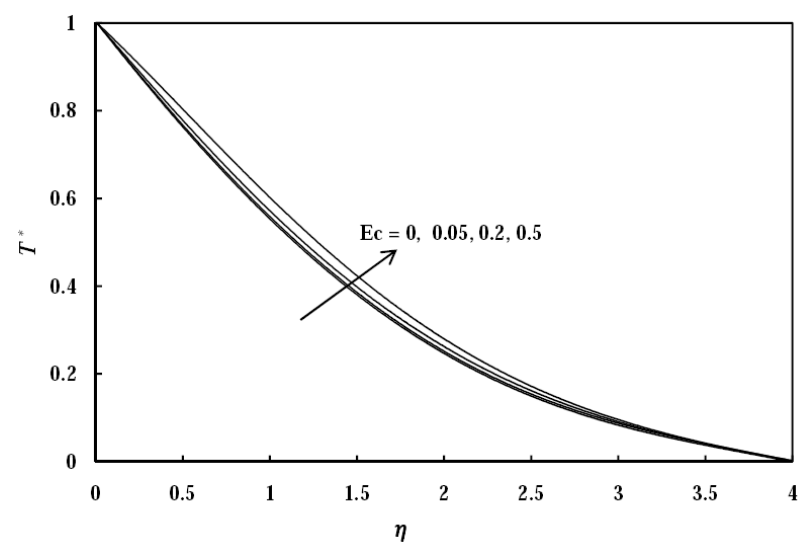

Fig. 7: Dimensionless temperature profile for different values of $\mathrm{Ec}$ when $\varepsilon=0.1, \mathrm{n}=0, \mathrm{M}=0.25$ and $\mathrm{Pr}=0.7$

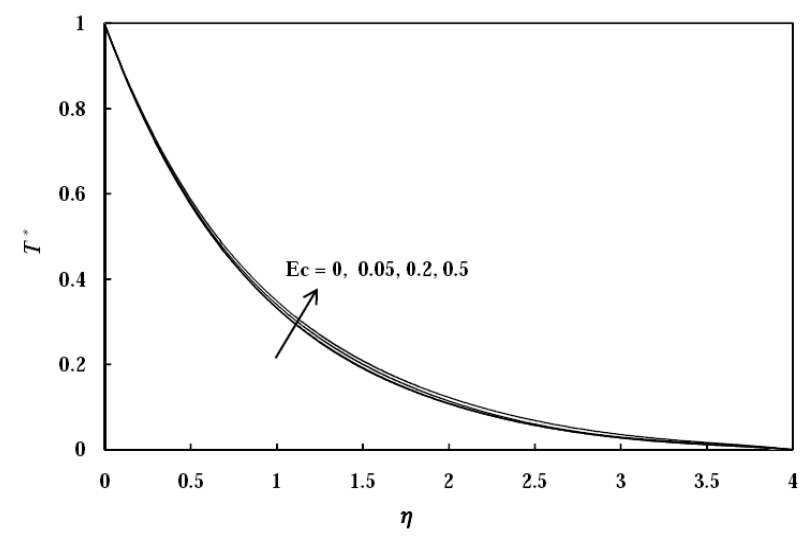

Fig. 8: Dimensionless temperature profile for different values of Ec when $\varepsilon=0.5, \mathrm{n}=2, \mathrm{M}=0.5$ and $\mathrm{Pr}=0.7$ 
From Figs. 9 and 10, it is found that the velocity gradient decreases with increasing values of the magnetic parameter $\mathrm{M}$ and the temperature gradient decreases for increasing values of $\mathrm{M}$.

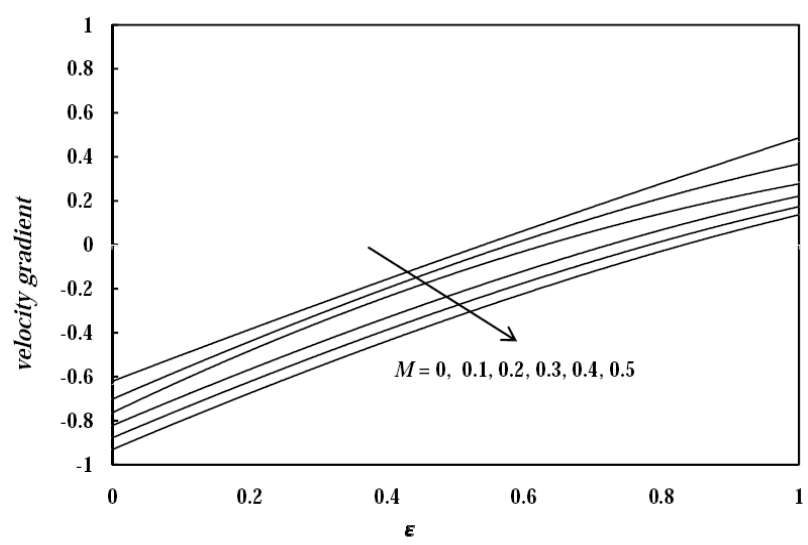

Fig. 9: Velocity gradient for different values of M

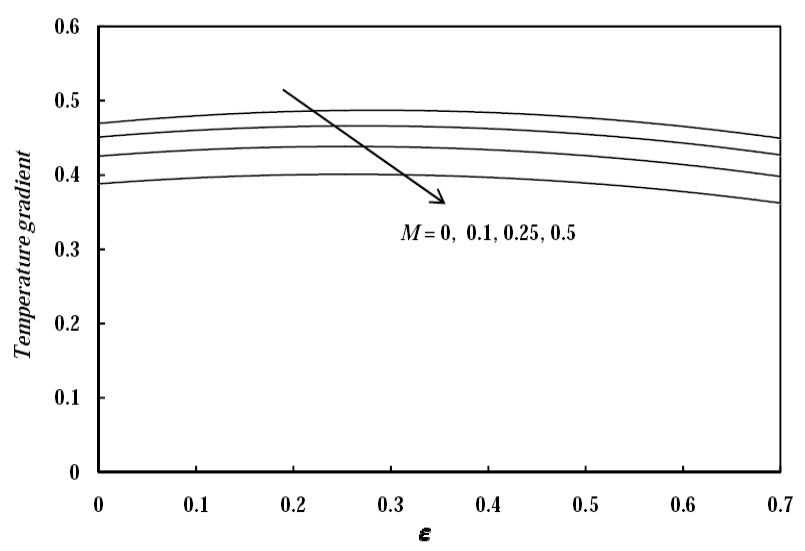

Fig. 10: Temperature gradient for different values of $M$ when $\operatorname{Pr}=0.7$

\section{Conclusion}

This work deals with the effects of magnetic parameter, moving parameter, viscous dissipation, skin friction and heat transfer over a moving nonisothermal flat plate. The governing partial differential equations are reduced to simple polynomial equations whose coefficients are of independent parameters $P_{r}, M, E c, \varepsilon, n$ and $R e$. These equations offer a practicing engineer a rapid way of obtaining shear stress and heat transfer for any combinations of $P_{r}, M, E c, \varepsilon, n$ and $R e$. The great advantage involved in the present technique is that the results are obtained with high order of accuracy and the amount of calculation is certainly less when compared with more conventional methods. Hence the practicing engineers and scientists can employ this unique approximate technique as a powerful tool for solving boundary layer flow and heat transfer problems.

\section{Acknowledgement}

The author wishes to thank the Management of SSN College of Engineering for providing the necessary facility to carry out the present work.

\section{References}

Afzal N, Badaruddin A, and Elgarvi AA (1933). Momentum and heat transport on a continuous flat surface moving in a parallel stream. International Journal of Heat and Mass Transfer, 36(13): 3399-3403

Blasius $H$ (1908). Grenschichten in flussigkeiten mit kleiner Reibung. Zeitschrift für Angewandte Mathematik und Physik, 56: 1-37.

Chandrasekar M (2003). Analytical study of heat transfer and boundary layer flow with suction and injection. Heat and Mass Transfer, 40 (1-2): 157-165.

Cortell R (2005). Numerical solutions of the classical Blasius flatplate problem. Applied Mathematics and Computation, 170(1): 706-710.

Erickson LE, Fan LT, and Fox VG (1966). Heat and mass transfer on moving continuous flat plate with suction or injection. Industrial and Engineering Chemistry Fundamentals, 5(1): 1925 .

Gyarmati I (1969). On the "governing principle of dissipative processes" and its extension to non-linear problems. Annalen der Physik, 478(7-8): 353-378.

Gyarmati I (1979). Non-equilibrium Thermodynamics: Field Theory and Variational Principles. Springer-Verlag, Berlin, Germany: 36-97.

Ishak N, Yacob NA, and Bachok N (2011). Radiation effects on the thermal boundary layer flow over a moving plate with convective boundary condition. Meccanica, 46(4): 795-801.

Jat RN and Neemawat A (2014). MHD boundary layer flow and heat transfer over a moving non-isothermal flat plate. Computational Mathematics and Modeling, 25(4): 514-520.

Kumari M and Nath G (2001). MHD boundary-layer flow of a nonNewtonian fluid over a continuously moving surface with a parallel free stream. Acta Mechanica, 146(3): 139-150.

Onsager L (1931a). Reciprocal relations in irreversible processes. I. Physical Review, 37(4): 405-406.

Onsager L (1931b). Reciprocal relations in irreversible processes. II. Physical Review, 38(12): 2265-2266.

Sakiadis BC (1961). Boundary layer behaviour on continuous solid surfaces: 1. Boundary-Layer Equations for Two-Dimensional and Axisymmetric Flow. American Institute of Chemical Engineers Journal, 7(1): 26-28.

Shanmugapriya M and Chandrasekar M (2008). Analytic solution of a free and forced convection with suction and injection over a non-isothermal wedge. Bulletin of the Malaysian Mathematical Sciences Society, Second Series, 31(1): 11-24.

Tsou FK, Sparrow EM, and Goldstein RJ (1967). Flow and heat transfer in the boundary layer on a continuous moving surface. International Journal of Heat and Mass Transfer, 10(2): 219-235. 